\title{
Investigación y desarrollo de un prototipo de Sistema Experto para Scheduling en Pymes con entorno Job Shop
}

\author{
Lindsay Álvarez Pomar ${ }^{*}$ \\ Camilo Caicedo Cárdenas** \\ Mario Malaver Gallego*** \\ Germán Méndez Giraldo***
}

\section{Resumen}

En el entorno competitivo actual, donde los cambios repentinos en la demanda y la apertura comercial obligan a las empresas a mantener un buen desempeño en todas sus áreas, la programación de tareas productivas (scheduling) de manera efectiva se ha vuelto no sólo un reto, sino una necesidad para sobrevivir en el mercado. Debido a que la mayoría de empresas en Colombia son pymes y cuentan con sistemas productivos tipo job shop, es importante prestar atención especial al job shop scheduling.

La complejidad del problema de job shop scheduling, sumada a la poca disponibilidad de personal especializado para esta labor, hacen necesario recurrir al empleo de herramientas computacionales, de alguna manera novedosas, que faciliten la función de scheduling. En esta investigación se creó una base de conocimiento y se diseñó una metodología para su implementación en un sistema experto para scheduling en pymes, con entorno job shop, cuyo empleo permite aumentar la efectividad en la toma de decisiones relacionadas con la gestión de la producción.

\section{Palabras Clave}

Job shop scheduling, sistemas expertos, pyme, gestión de la producción.

DESIGN OF SYSTEM EXPERT PROTOTYPE TO SCHEDULING IN JOB-SHOP ENVIRONMENT

* Grupo de Investigación Sistemas Expertos y Simulación - SES Universidad Distrital Francisco José de Caldas. lalvarez@udistrital. edu.co

* Investigador Auxiliar Grupo SES camilo.caicedo@gmail.com

*** Investigador Auxiliar Grupo SES mario.malaver@gmail.com

****Investigador Principal y co-director Grupo SES gmendez@udistrital.edu.co 


\section{Abstract}

In the current competitive environment, where both sudden changes in products demand and economic globalization demand every area of the organization to keep a good performance, the effective scheduling has become not only a challenge, but also a necessity to survive within the marketplace. Due to the fact that most of Colombian enterprises are SMEs and most of them have their productive systems set up as job shop, it becomes important to pay special attention to job shop scheduling.

Job shop scheduling problem complexity, in addition to low availability of specialized staff to perform this task, make necessary to resort to the use of computational tools, somehow innovative, which facilitate the scheduling function. In order to tackle this problem, through this research has been created a knowledge base and has been designed a methodology for its implementation into an expert system for job shop scheduling, which is applicable to the SMEs.

\section{Keywords}

Job Shop Scheduling, expert systems, SME, production management.

\section{Introducción}

El scheduling es una forma de toma de decisiones que juega un rol crucial en las industrias de manufactura y servicios. En el entorno competitivo actual, donde los cambios repentinos en la demanda y en la apertura comercial obligan a las empresas a mantener un buen desempeño en todas sus áreas, el scheduling efectivo se ha vuelto no sólo un reto, sino una necesidad para sobrevivir en el mercado.

Las pymes, en especial aquellas que buscan un crecimiento sostenible, deben realizar scheduling. La razón principal para prestar atención es su im- portancia en el control del costo de producción, en la calidad del producto y en la satisfacción del cliente - así como en la cantidad de tiempo que las firmas dedican, rutinariamente, a la realización de esta función.

En el caso de las pymes que no realizan scheduling, se presentan problemas como: conflictos entre recursos, aumento del inventario de producto en proceso, tiempos ociosos de maquinaria y personal, retrasos en los tiempos de entrega, insatisfacción del cliente y pérdida de la cuota de mercado. Todo esto conlleva a una disminución en los ingresos percibidos por la empresa y, a la vez, elevan los costos de producción (afectando negativamente la rentabilidad de la misma).

Para el caso particular de la pequeña y mediana empresa pyme en Colombia, el $71.5 \%$ realizan scheduling y tan sólo en el 1.8\% confiere éste cargo a programadores de la producción (Méndez y Álvarez, 2004). Lo anterior es una causa para que algunas compañías no sean tan competitivas como lo podrían ser; al no realizar un scheduling apropiado se obtiene, como consecuencia, un manejo ineficiente de los recursos, retrasos en los tiempos de entrega, pérdida significativa de good will, insatisfacción de los requerimientos del cliente, etc.

$\mathrm{Al}$ analizar la cifra, significativamente baja, correspondiente al porcentaje de actividades de scheduling llevadas a cabo por programadores, se sugieren como justificaciones de ello la subestimación de la importancia del scheduling en la gestión efectiva de la producción y los altos costos asociados a la contratación de un programador de la producción (scheduler) —entendiendo éste como una persona con alto grado de especialización en dicha labor- debido a su escasez y poca disponibilidad. Sumado a esto, realizar scheduling en un entorno job shop, que corresponde al 70,3\% de las pymes en nuestro país (Méndez y Álvarez, 2004), es una tarea de alto grado de dificultad ya que es un problema de optimización combinatoria.

En esta investigación se busca atacar la causa asociada a los altos costos de contratación y a la poca 
disponibilidad de un programador especializado. Con éste fin se creó una base de conocimiento y se diseñó una metodología para su implementación en un sistema experto para scheduling en pymes, con entorno job shop, cuyo empleo permita aumentar la efectividad en la toma de decisiones relacionadas con la gestión de la producción.

En la sección 2 se presentan brevemente los conceptos relacionados con la gestión de la producción y sistemas expertos. Posteriormente, en la sección 3 se expone la metodología diseñada y la manera en que ésta fue abordada para el desarrollo del prototipo. Las secciones 4 y 5 contienen tanto los resultados como las conclusiones de la investigación, respectivamente. Finalmente, en la sección 6 se proponen posibles trabajos a futuro basados en este trabajo.

\section{Contexto teórico}

\section{Gestión de la producción}

La administración de un sistema productivo responde a un proceso jerárquico de toma de decisiones que está soportado en las estrategias de la compañía. Este proceso es denominado gestión de la producción y sus principales etapas son: $a$ ) planeación estratégica; $b$ ) planeación agregada; y $c$ ) scheduling. En las dos primeras se toman decisiones que permiten hacer disponibles los recursos; mientras que en el scheduling se toman decisiones sobre la asignación de tales recursos.

\section{Scheduling}

Se puede definir scheduling como un proceso crítico de toma de decisiones — en el quehacer de una organización-que optimiza la asignación de recursos disponibles en función del cumplimiento de los objetivos de la organización y de los compromisos que ésta ha adquirido con sus clientes.

\section{Job Shop}

En este tipo de sistema, también conocido como process layout (disposición por proceso), cada trabajo tiene rutas diferentes a través del sistema (ver figura núm.. 1) y transitan en lotes, de una operación a otra, almacenándose temporalmente en cada una de las máquinas $(m)$ en espera de su turno (Buffa, 1980; Askin y Goldberg, 2002). Las instalaciones de manufactura pueden ser organizadas de acuerdo a los procesos - de manufacturainvolucrados en la producción de su portafolio de productos. En dicha organización, la maquinaria es agrupada al interior de la planta basándose en propósitos comunes: por ejemplo, un grupo de tornos, trilladoras, etc. Desde el punto de vista de la variedad de productos, la disposición por proceso es la organización de la producción más flexible; sin embargo, tiene la desventaja de ser la más difícil para controlar (Buffa y Sarin, 1992; Elsayed y Boucher, 1994; Chase, Aquilano y Jacobs, 2001; Méndez Giraldo, 2003) y sufre de una baja utilización del equipamiento y largos tiempos de flujo (Askin y Goldberg, 2002).

\section{FIGURA 1. Sistema productivo tipo Job Shop. Tomado y modificado de Asking \& Goldberg (2002).}

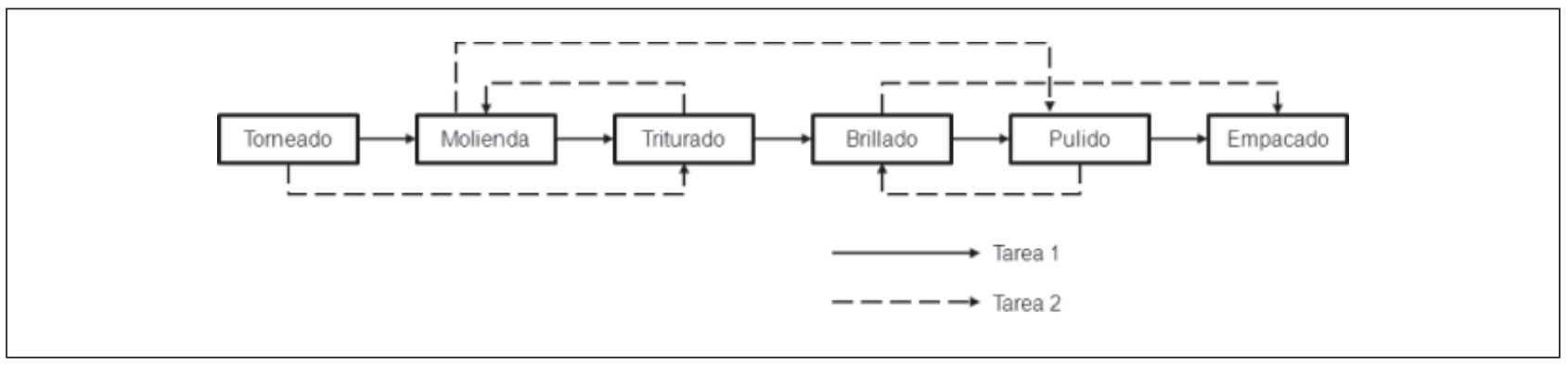




\section{Job Shop Scheduling}

En el caso del Job Shop, las tareas necesitan ser enrutadas mediante centros de trabajo organizados funcionalmente. Cuando un trabajo llega a un centro de labores, ingresa en una fila y espera, por medio de una máquina disponible, para realizar la operación que requiere. Scheduling, en este caso, involucra determinar el orden para la ejecución de los trabajos y la asignación de una máquina para efectuar la operación requerida (Chase, Aquilano y Jacobs, 2001).

\section{Sistema Experto}

Un Sistema Experto (SE) es un programa de computador que tiene una amplia base de conocimiento en un dominio específico y emplea técnicas de inferencia computacional para tratar con problemas usualmente resueltos por humanos expertos. Este programa simula el proceso de aprendizaje, memorización, razonamiento, comunicación y acción del experto.

El SE no pretende reproducir exactamente el modus operandi de un hombre. Éste último es frecuentemente sobrecargado por su trabajo diario y, en ciertas ocasiones, no cuenta con el tiempo necesario para evaluar las reglas más apropiadas. Además, el SE tiene una capacidad de procesamiento y cálculo que excede la capacidad humana - esto puede ser usado para mejorar la tarea del experto humano.

El proceso de construcción de un SE se denomina ingeniería del conocimiento y consiste en la adquisición de saberes a partir de un especialista humano y mediante extensas entrevistas con un ingeniero del conocimiento $\longrightarrow$ o de otra fuente- para su posterior codificación en un esquema de representación del conocimiento (esto le permitirá, al sistema, manipular el conocimiento almacenado para buscar soluciones). En la figura núm. 2, se presenta la estructura general de un SE.

Base de conocimiento: contiene el discernimiento necesario para resolver un problema específico.

\section{FIGURA 2: estructura de un Sistema Experto.}

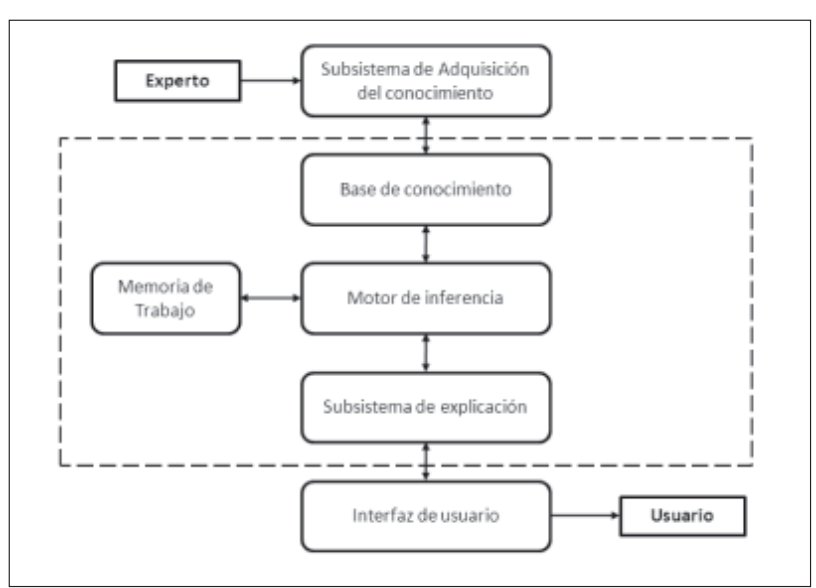

Motor de inferencia: contiene los algoritmos para deducir conclusiones o soluciones fundamentadas en el conocimiento de la base.

Módulo de explicación: se encarga de justificar y explicar el razonamiento producido por el Sistema Experto en la solución de un problema específico.

Memoria de trabajo: es un área reservada en la memoria del computador para guardar el registro de las entradas, las conclusiones dentro del proceso y las salidas finales.

Interfaz con el usuario: éste componente es la forma en la que el sistema interactúa con el usuario.

\section{Desarrollo del Prototipo}

La construcción del prototipo se realizó de acuerdo con las siguientes fases:

Fase 1: adquisición del conocimiento.

Fase 2: elección de la herramienta de desarrollo.

Fase 3: construcción del prototipo.

Fase 4: validación del prototipo.

El desarrollo del prototipo se llevó a cabo al interior del Grupo de Investigación Sistemas Expertos y Simulación (Gises). El equipo encargado del 
proyecto estuvo conformado por: Ph.D Germán Andrés Méndez Giraldo, MSc. Lindsay Álvarez Pomar; y los estudiantes de ingeniería industrial Camilo Andrés Caicedo Cárdenas y Mario Andrés Malaver Gallego.

\section{Adquisición del conocimiento}

En esta fase se identificaron las piezas claves del conocimiento (conceptos, reglas y estrategias) las cuales permitieron establecer las teorías sobre la organización del mismo y las estrategias de resolución del problema de scheduling. Se desarrolló en tres etapas: adquisición del conocimiento del experto, adquisición del conocimiento adicional y formulación del conocimiento sintético.

\section{- Adquisición del conocimiento del experto}

Para el desarrollo de esta investigación ofició como experto el Ph.D Germán Andrés Méndez Giraldo. En la adquisición del conocimiento de esta fuente se identificaron, por medio de discusiones abiertas (brainstorming) y debates al interior del grupo de trabajo — dirigidas por el experto-, los aspectos que influyen en la toma de decisiones en la función de scheduling.

Estos aspectos se analizaron y se estructuraron en un diagrama de Ishikawa (espina de pescado) relacionando los principales factores influyentes en la función de scheduling con sus respectivas variables (ver figura núm. 3).

\section{FIGURA 3: aspectos influyentes en la función de scheduling.}

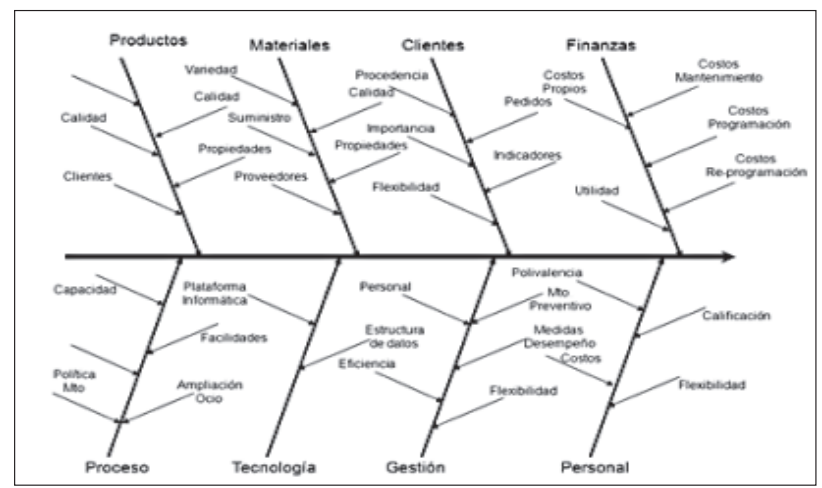

FIGURA 4: extracto de diseño de entrevista para el factor producto.

\begin{tabular}{|c|c|c|c|}
\hline \multicolumn{4}{|c|}{ FACTOR: PRODUCTO } \\
\hline & Pregunta & ¿Paraqué? & Medida \\
\hline \multicolumn{4}{|c|}{ Caracteristicas y propiedades de los productos } \\
\hline 3 & $\begin{array}{l}\text { ¿En la empresa se } \\
\text { fabrican productos } \\
\text { perecederos? }\end{array}$ & $\begin{array}{l}\text { Si se fabrican productos perecederos, es muy } \\
\text { importante tener en cuenta el tiempo máximo } \\
\text { que estos productos se pueden tener en } \\
\text { inventario y el costo en que se incurre al } \\
\text { tenerlos alli, para elegir la medida de } \\
\text { desempeño adecuadaalhacer la programación. }\end{array}$ & ${ }^{51}{ }^{\text {No }}$ \\
\hline
\end{tabular}

Posteriormente, se utilizó como metodología el diseño de entrevistas dirigidas a los encargados de la programación de la producción en las pymes (ver figura número. 4) con el fin de determinar qué información requiere el experto para la toma de decisiones en la función de scheduling. El diseño de estas entrevistas se obtuvo a partir de sesiones de socialización y discusión al interior del grupo de trabajo. Por su parte, el resultado del proceso constituyó la base para la formulación del conocimiento sintético.

\section{- Adquisición del conocimiento adicional}

En esta fase se consultaron fuentes de conocimiento secundarias tales como papers técnicos y literatura especializada con el objetivo de ampliar el conocimiento sobre los métodos existentes para la solución al problema de Job Shop Scheduling y su desempeño de acuerdo a diferentes configuraciones de sistemas productivos. El análisis comparativo del desempeño de las técnicas de solución más comunes se presenta en la Tabla No. 1.

\section{- Formulación del conocimiento sintético}

En base a los resultados obtenidos durante los procesos de adquisición del conocimiento, y bajo la dirección del experto, se estructuró el conocimiento sintético formulando piezas de conocimiento explícito del dominio de Job Shop Scheduling (ver Figura No. 5) a partir de las cuales se generaron las reglas que posteriormente fueron implementadas en la base de conocimiento.

Este conocimiento sintético estuvo sujeto a modificaciones y complementaciones por medio de un proceso iterativo entre las etapas de construcción y validación del prototipo del Sistema Experto. 
TABLA 1: técnicas de solución para Job Shop Scheduling.

\begin{tabular}{|c|c|c|c|}
\hline \multicolumn{2}{|c|}{ TECNICA } & VENTAJAS & DESVENTAJAS \\
\hline \multirow{3}{*}{\multicolumn{2}{|c|}{ Formulación Matemática }} & \multirow{3}{*}{ Los problemas pueden ser resueltos óptimamente. } & $\begin{array}{l}\text { Sólo son capaces de resolver instancias al tamente simplificadas } \\
\text { en un lapso de tiempo razonable. }\end{array}$ \\
\hline & & & El número de variables enteras crece exponencialmente. \\
\hline & & & $\begin{array}{l}\text { Una formulación de programación entera para problemas de } \\
\text { Scheduling es computacionalmente infactible. }\end{array}$ \\
\hline \multirow{3}{*}{$\begin{array}{l}\text { Técnicas de } \\
\text { Aproximación }\end{array}$} & \multirow{3}{*}{$\begin{array}{l}\text { Reglas de } \\
\text { Prioridad }\end{array}$} & $\begin{array}{l}\text { La facilidad de su implementación y su requerimiento } \\
\text { computacional. }\end{array}$ & $\begin{array}{l}\text { Los resultados se logran muy rápido pero se pueden desviar } \\
\text { hasta un } 74 \% \text { del óptimo y en general la calidad de la solución } \\
\text { se degrada en la medida en que la dimensionalidad del } \\
\text { problemase incrementará. }\end{array}$ \\
\hline & & \multirow[b]{2}{*}{$\begin{array}{l}\text { Las reglas de prioridad son más convenientes como una } \\
\text { técnica para solución inicial que para ser consideradas como } \\
\text { alternativas de solución del JSP. }\end{array}$} & $\begin{array}{l}\text { Las reglas individuales se desempeñan pobremente y no } \\
\text { proveen ninguna conclusión con respecto al makespon. }\end{array}$ \\
\hline & & & $\begin{array}{l}\text { Cuando se usan procedimientos para mejorar las selecciones } \\
\text { hechas por las reglas de prioridad, los tiempos } \\
\text { computacionales son } 3 \text { veces más grandes con respecto a las } \\
\text { reglas de prioridadindividuales. }\end{array}$ \\
\hline \multirow{5}{*}{$\begin{array}{l}\text { Técnicas de } \\
\text { Búsqueda Local }\end{array}$} & \multirow{2}{*}{ Búsqueda Tabú } & $\begin{array}{l}\text { Proveen buenas soluciones y ofrecen posibilidades de ser } \\
\text { potenciados cuando se combinan con otras heurísticas. }\end{array}$ & \multirow{2}{*}{$\begin{array}{l}\text { Requiere que muchos parámetros sean cuidadosamente } \\
\text { refinadosy apropiadamente ajustados para cada problema. }\end{array}$} \\
\hline & & $\begin{array}{l}\text { En general TS provee los mejores resultados entre todas las } \\
\text { técnicas. }\end{array}$ & \\
\hline & $\begin{array}{l}\text { Recocido } \\
\text { Simulado }\end{array}$ & $\begin{array}{l}\text { Proveen buenas soluciones y ofrecen posibilidades de ser } \\
\text { potenciados cuando se combinan con otras heurísticas. }\end{array}$ & $\begin{array}{l}\text { El excesivo tiempo computacional necesario para alcanzar } \\
\text { buenas soluciones. }\end{array}$ \\
\hline & \multirow[t]{2}{*}{$\begin{array}{l}\text { Algoritmos } \\
\text { genéticos }\end{array}$} & \multirow[t]{2}{*}{$\begin{array}{l}\text { Proveen buenas soluciones y ofrecen posibilidades de ser } \\
\text { potenciados cuando se combinan con otras heurísticas. }\end{array}$} & $\begin{array}{l}\text { No son muy apropiados para poner a punta estructuras que } \\
\text { están muy cerca de las soluciones óptimas en la medida que } \\
\text { los operadores de cruce generalmente pierden su eficiencia } \\
\text { paragenerar Schedules factibles. }\end{array}$ \\
\hline & & & $\begin{array}{l}\text { Dan pobre resultados debido a las dificultades que tienen con } \\
\text { los operadores de cruce y la codificación de Schedules. }\end{array}$ \\
\hline \multirow{2}{*}{\multicolumn{2}{|c|}{ Simulación }} & Es una herramienta de análisis flexible y poderosa. & \multirow[b]{2}{*}{ Requiere una gran cantidad de conocimiento. } \\
\hline & & $\begin{array}{l}\text { Puede ayudar a evaluar si los Schedules van a trabajar bien } \\
\text { bajo condiciones reales, identificando las mejores en la } \\
\text { planta. }\end{array}$ & \\
\hline \multicolumn{2}{|c|}{ Teoría de Restricciones } & $\begin{array}{l}\text { Permite reducir la dimensión de los problemas de } \\
\text { Scheduling al contemplar principalmente los recursos que } \\
\text { restringen elflujo. }\end{array}$ & $\begin{array}{l}\text { Como es una filosofía, su implementación requiere de cambios } \\
\text { importantes en la organización. }\end{array}$ \\
\hline
\end{tabular}

\section{FIGURA 5: aspectos influyentes en la función de scheduling.}

Restricciones del producto: Se puede 0 no almacenar, las condiciones físico-químicas modifican las decisiones de tener inventario, tienen fechas de vencimiento, son perecederos, hay en la misma organización la posibilidad de sustituir productos unos con otros.

\section{Elección de la herramienta de desarrollo}

Se eligió Clips como herramienta de desarrollo teniendo en cuenta los siguientes aspectos:

\section{- Representación del conocimiento}

El conocimiento de un experto humano es heurístico (basado en la experiencia). Clips utiliza las reglas para representar heurísticas que especifican un conjunto de acciones a ser realizadas para una situación dada. Por lo tanto, es conveniente representar el conocimiento del experto por medio de reglas de producción. Ésta herramienta, además, emplea las reglas como uno de los principales métodos de representación del conocimiento.

\section{- Disponibilidad y costo}

Clips es un software de dominio público, por lo tanto no tiene ningún costo y su versión más reciente se encuentra disponible en: www.ghg.net/ clips/Download.html.

REVISTA CIENTÍFICA / ENERO -DICIEMBRE DE 2010 / No. 12 / BOGOTÁ, D.C. 


\section{- Compatibilidad con otros lenguajes}

El programa puede ser encajado dentro de un código procedimental, llamado como una subrutina e integrado con lenguajes como C y Java.

\section{- Requisitos computacionales}

Clips está escrito en C para mayor portabilidad y velocidad y ha sido instalado en muchos sistemas operativos sin necesidad de realizar cambios de código.

\section{Construcción del prototipo}

La construcción del prototipo se realizó en dos pasos:

- Creación de reglas y diseño de la base de conocimiento

A partir del conocimiento sintético se formularon reglas de producción de tipo "SI- ENTONCES", las cuales fueron utilizadas para la creación de la base del conocimiento del prototipo del Sistema Experto. Estas reglas están representadas en forma de árbol de decisión (figura No. 6).

\section{- Implementación por medio de la herramienta seleccionada}

Las reglas contenidas en el árbol de decisión se programaron en Clips utilizando hechos y reglas,

\section{FIGURA 6: segmento del árbol de decisión (módulo} de restricciones).

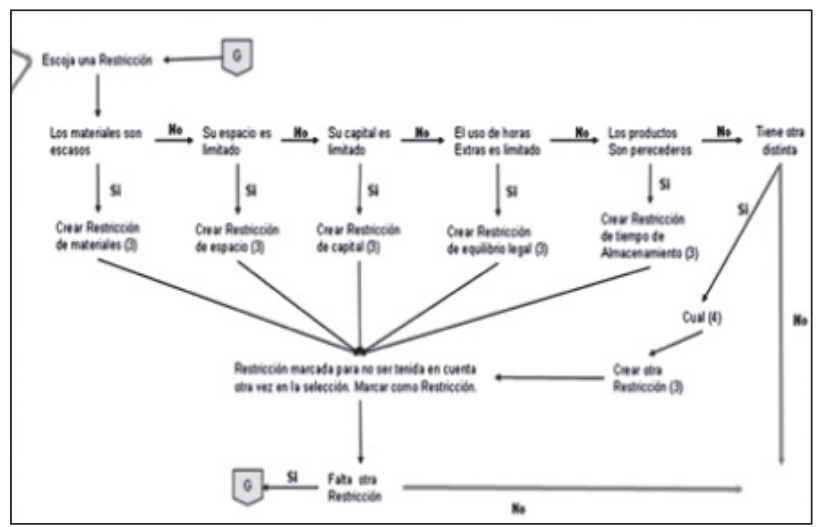

\section{FIGURA 7: proceso de ingeniería del conocimiento.}

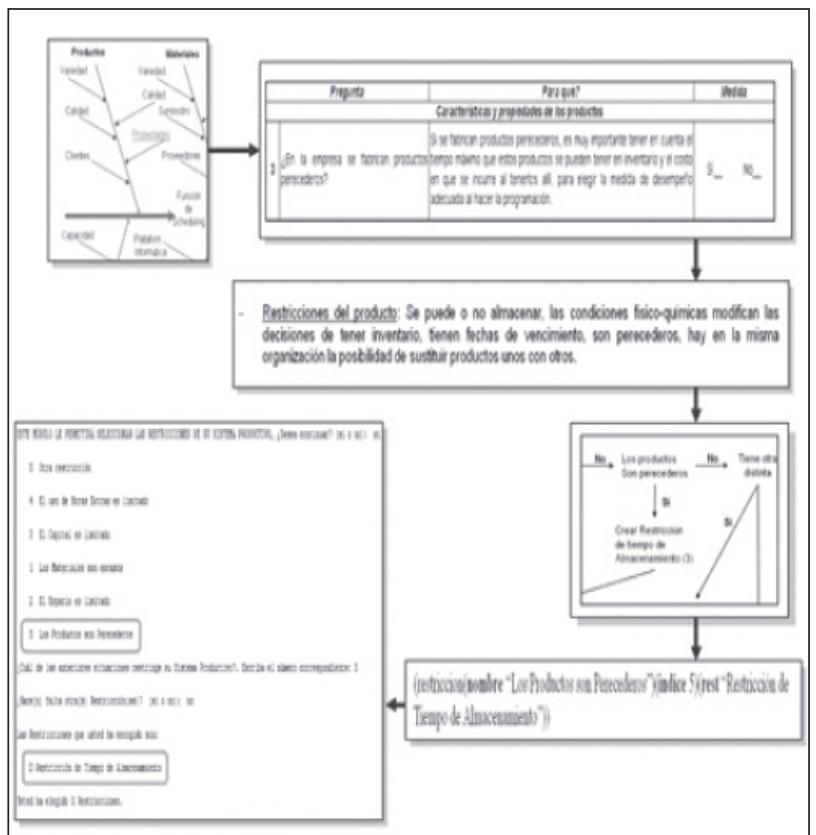

principalmente. Un hecho es cada fragmento de información que un programa de Clips requiere para razonar con el fin de resolver problemas; mientras que una regla de tipo "SI-ENTONCES" es un método para representar el conocimiento en un Sistema Experto. La figura No. 7 expone el proceso realizado para codificar el conocimiento adquirido de manera que pueda ser implementado en Clips.

En la Tabla No. 2 se muestra la estructura del prototipo:

\section{Validación del prototipo}

El principal objetivo de esta fase fue validar la estructura general y la base de conocimiento del prototipo. Esta etapa se desarrolló de forma iterativa durante de la construcción del prototipo, cumpliendo, así, el ciclo implementación-mejora tantas veces como fuera necesario para obtener la satisfacción del experto. Este ciclo se aplicó en dos aspectos: validación de la base del conocimiento y validación de la programación del prototipo a través de la evaluación de escenarios y pruebas de software, respectivamente. 


\section{TABLA 2: módulos del prototipo.}

\begin{tabular}{|c|c|c|}
\hline Nombre Módulo & Función & $\begin{array}{l}\text { No. } \\
\text { reglas }\end{array}$ \\
\hline Módulo Inicial & $\begin{array}{l}\text { Este módulo permite obtener la } \\
\text { información necesaria para inicializar el } \\
\text { programa. }\end{array}$ & 8 \\
\hline $\begin{array}{l}\text { Módulo para determinar } \\
\text { el tipo de Sistema } \\
\text { Productivo. }\end{array}$ & $\begin{array}{l}\text { Este módulo permite determinar si se trata } \\
\text { de un sistema tipo Job Shop para poder } \\
\text { continuar con la ejecución del programa. }\end{array}$ & 8 \\
\hline $\begin{array}{l}\text { Módulo para seleccionar } \\
\text { la(s) Médida(s) de } \\
\text { Desempeño. }\end{array}$ & $\begin{array}{l}\text { Este módulo permite seleccionar una } \\
\text { medida de desempeño. Este módulo se } \\
\text { activa sólo si el usuario sabe cuál medida } \\
\text { de desempeño es apropiada para su } \\
\text { Sistema Productivo. }\end{array}$ & 21 \\
\hline $\begin{array}{l}\text { Módulo para definir la(s) } \\
\text { Médida(s) de } \\
\text { Desempeño. }\end{array}$ & $\begin{array}{l}\text { Este módulo permite definir una medida de } \\
\text { desempeño según las preferencias del } \\
\text { usuario. }\end{array}$ & 56 \\
\hline $\begin{array}{l}\text { Módulo para ponderar } \\
\text { la(s) Médida(s) de } \\
\text { Desempeño. }\end{array}$ & $\begin{array}{l}\text { Este módulo permite asignar pesos a cada } \\
\text { una de las medidas de desempeño } \\
\text { seleccionadas. }\end{array}$ & 9 \\
\hline $\begin{array}{l}\text { Módulo para establecer } \\
\text { la(s) Restricción(es) del } \\
\text { Sistema Productivo. }\end{array}$ & $\begin{array}{l}\text { Este módulo permite crear las } \\
\text { restricciones particulares de cada Sistema } \\
\text { Productivo. }\end{array}$ & 22 \\
\hline $\begin{array}{l}\text { Módulo para definir la } \\
\text { técnica de Scheduling } \\
\text { apropiada. }\end{array}$ & $\begin{array}{l}\text { Este módulo permite determinar la técnica } \\
\text { a utilizar para resolver el problema de } \\
\text { Scheduling según las caracteristicas del } \\
\text { Sistema Productivo, sus restricciones y las } \\
\text { medidas de desempeño consideradas. }\end{array}$ & 12 \\
\hline
\end{tabular}

\section{Validación de la base de conocimiento}

Se desarrolló a través de la evaluación del cumplimiento de las reglas en la solución de diferentes escenarios supuestos - procurando evaluar la mayor cantidad de situaciones posibles. Es decir, se supusieron condiciones muy particulares de sistemas productivos para ratificar el funcionamiento de cada una de las reglas. Como resultado de este proceso la regla fue validada, modificada, eliminada, sustituida o complementada con otra(s) regla(s).

\section{Validación de la programación del prototipo}

Para la validación de la programación del prototipo se realizaron las siguientes pruebas:

\section{- Inspección manual del código:}

Se realizaron lecturas línea a línea del código verificando que la sintaxis fuera correcta y coherente.

\section{- Prueba de ejecución}

Consistió en ir ejecutando el código para verificar su funcionamiento. En los casos en que algún módulo falló, se utilizó un depurador para observar la evolución dinámica del sistema, localizar el fallo, y repararlo. Para realizar esta depuración se utilizó el comando de Clips "watch all".

Pruebas de caja blanca: se ejecuta el programa conociendo el código. Se procuró recorrer todos los caminos posibles que "debería" tomar el programa cuando se está ejecutando para verificar el funcionamiento correcto de las reglas.

Pruebas de caja negra: se ejecuta el programa sin previo conocimiento del código (limitándose a la interfaz). En estas pruebas se intentó descubrir casos y circunstancias en los que algún módulo no hiciera lo que se espera de éste.

\section{Pruebas de robustez}

La capacidad del sistema, para salir de situaciones provocadas por errores en el suministro de datos, fue puesta a prueba. Por ejemplo, se ensayaron los siguientes casos: órdenes con defectos de sintaxis, datos erróneos, ingreso de valores incorrectos y órdenes nulas (líneas vacías).

\section{Resultados}

A partir de esta investigación se obtuvieron los siguientes resultados: cuerpo de conocimiento experto sobre Job Shop Scheduling, metodología para la construcción de un Sistema Experto basado en reglas y el prototipo del Sistema Experto para scheduling en pymes con entorno Job Shop.

A. Cuerpo de conocimiento experto: el conocimiento sintético y las reglas representadas en el árbol de decisión contienen conocimiento experto aplicable a la toma de decisiones en problemas relacionados con Job Shop Scheduling. Este resultado sirve como marco de referencia para la realización de otros proyectos de investigación en esta área de conocimiento. 
b. Metodología para la construcción de un Sistema Experto: permite transformar el conocimiento abstracto, por medio de la identificación de relaciones lineales entre variables, en piezas de conocimiento explícito, articularlas en una base de conocimiento e implementar ésta en un Sistema Experto para solucionar un problema específico. Esta metodología se muestra en la Figura No. 8 y puede ser adoptada para facilitar el desarrollo de un Sistema Experto en cualquier dominio de conocimiento.

c. Prototipo del Sistema Experto: el prototipo construido permite, según las condiciones particulares y los objetivos de cada pyme, sugerir la técnica y la(s) medida(s) de desempeño más conveniente(s) para realizar scheduling. De esta manera, cuando el prototipo se convierta en un software completamente utilizable, permitirá mejorar la toma de decisiones relacionadas con esta función. En la Figura No. 9 es explaneado un pantallazo de la ejecución del software.

\section{Conclusiones}

Un Sistema Experto puede, según las condiciones particulares y los objetivos de cada pyme, sugerir la técnica y la(s) medida(s) de desempeño más conveniente(s) para realizar scheduling. Esto permite al "no-experto" aplicar las técnicas y las medidas de desempeño que el programador experto aplicaría.

Existen diversas técnicas de scheduling y diferentes configuraciones de sistemas productivos con condiciones particulares. Se requiere experticia, ya sea proveniente de un software o de un experto humano, para decidir qué técnica aplicar en un sistema productivo dado.

Es posible crear una metodología robusta para la construcción de un Sistema Experto basado en reglas que permita:

- Transformar el conocimiento abstracto, por medio de la identificación de relaciones li-

\section{FIGURA 8: metodología diseñada para la construcción del Sistema Experto.}

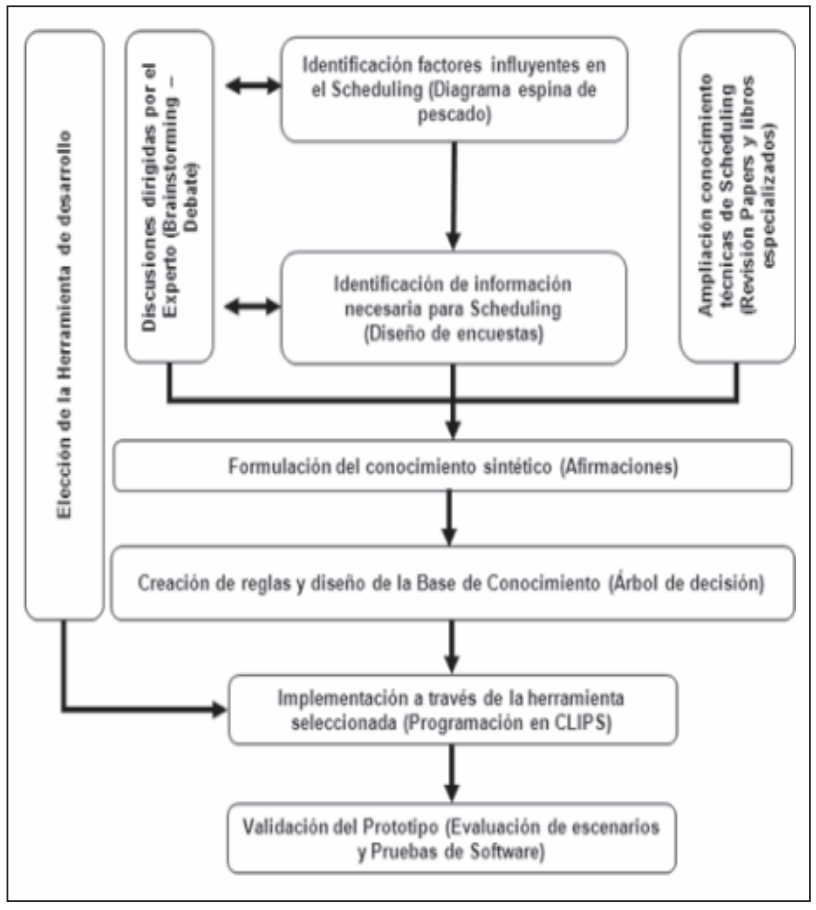

\section{FIGURA 9: vista de la ejecución del Sistema Experto.}

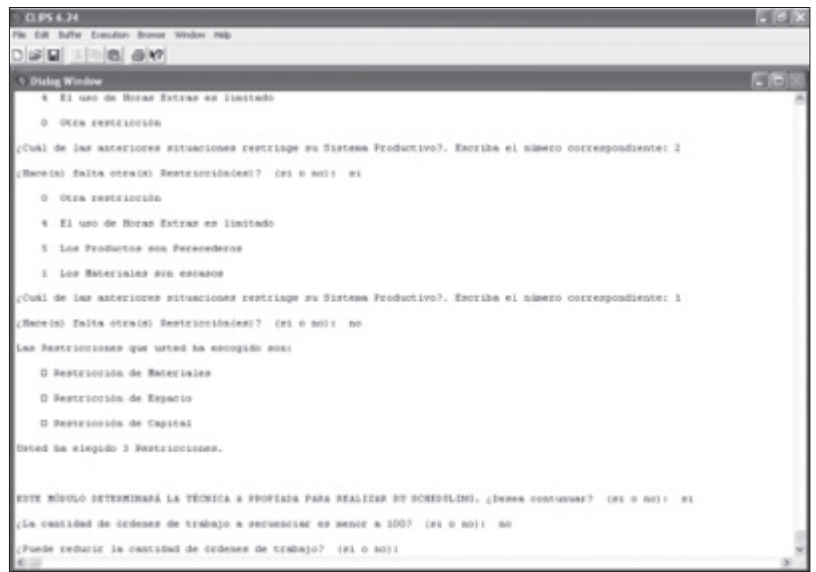

neales entre variables, en piezas de conocimiento explícito.

- Articular estas piezas en una base de conocimiento.

- Implementar esta base de conocimiento en un Sistema Experto para solucionar un problema específico. 


\section{Trabajos futuros}

A partir de este trabajo, se pretende llevar a cabo investigaciones posteriores que permitan:

- La complementación del Sistema Experto con un módulo que ejecute las técnicas sugeridas.

- El refinamiento del prototipo para convertirlo en software plenamente utilizable.

- La inclusión del Prototipo de Sistema Experto para Scheduling dentro del proyecto Sistema Integral de diagnóstico para la pyme (proyecto macro del Grupo de Investigación Sistemas Expertos y Simulación).

\section{Bibliografía}

- Adam, E. y Ebert, R. (1991), Administración de la producción y las operaciones, México D.F., Prentice Hall.

-Al-Ahmari, A. (2002), "Optimización computarizada de secuenciación y parámetros de maquinado en un sistema de manufactura tipo Job Shop", en Paper

—Andriani, C.; Biasca, R. y Rodríguez, M. (2003), Un nuevo sistema de gestión para lograr PYMES de clase mundial, México D.F., Grupo Editorial Norma

- Askin, R. y Goldberg, J. (2002), Design and Analysis of Lean Production Systems,

— Bagchi, T. (1999), Multiobjective Scheduling by genetic algorithms, Kluwer Academic Publishers.

- Barriga, E. (2006, marzo) "Pyme, importancia nacional e internacional", en Caja de herramientas para las Pymes, núm. 1.

— Buffa, E. (1980), Administración de operaciones, México D.F., Limusa Editores.
- Buffa, E. y Sarín, R. (1992), Administración de la producción y de las operaciones, México D.F., Limusa Editores.

- Cardona, M.; Barriga, E. y Cano, C. (2006, junio), "Mejoramiento de la competitividad en las Pymes colombianas" en Caja de herramientas para las Pymes, núm. No. 27.

- Chase, R.; Aquilano, N. y Jacobs, R. (2001), Operations Management for Competitive Advantage. New York, Mc Graw Hill.

— CHIU, W.C. (2000), "An Overview of Solving Job Shop Scheduling Problem by Local Search Techniques", en Paper.

— Comesaña, J. y Prado, C. (2002), "Creating an Expert System for detailed Scheduling", en $\mathrm{Pa}$ per International of Operations \& Production Management, vol. 22, núm. 7, p. 806.

- Corredor, M. (2000), Principios de Inteligencia Artificial \& Sistemas Expertos, Bucaramanga, Universidad Industrial de Santander.

— Durkin, J. (1994), Expert Systems: Design and Development, New Jersey, Prentice Hall.

— Elsayed, E. y Boucher, T. (1994), Analysis and control of Production Systems, New Jersey, Prentice Hall.

—Engelmore, R. y Feigenbaum, E. (2000), "Expert Systems and Artificial Intelligence" [en línea], disponible en: http://www.wtec.org/loyola/ kb/c1_s1.htm, recuperado: 1 septiembre de 2000 .

- Ghrayeb, O. () "An Efficient Genetic Algorithm for Job-Shop Scheduling Problems with Imprecise Processing Times", en Paper Department of Industrial Engineering. University.

— Giarratano, J. y Riley, G. (2001), Sistemas Expertos. Principios y Programación, México D.F., International Thomson Editores. 
- Gibson, D. (2001, junio), “To Schedule or not to Schedule”, en Paper, tomo 33, núm. 6, p. 33.

Gómez y Casanovas (2000). "Use of Agents and Simulation in Production Scheduling", en Paper

- Jackson, P. (1990) Introduction to Expert Systems, Wesley, Addison.

— Jain, A.S. y Meeran, S. (1998), "A State-of-theart review of Job-Shop Scheduling Techniques", en Paper

— Jones, A. y Rabelo, L.C. (1998), "Survey of Job Shop Scheduling Techniques”, en Paper

— Kiong, S.W. (2005), "MobES Expert System" [en línea], dsponible en: http://www.generation5. org/content/2005/MobES.asp, recuperado: 1 de junio.

—Land, S.M. (2006), "Expert System Design Shells: A Critical Analysis" [en línea], disponible en: http://www2.gsu.edu/ wwwitr/docs/esshells/ index.html, recuperado: 1 de octubre.

— Lang-Fang, H.; Ross, P. y Corne, D. (193), “A promising GA Approach to Job-Shop Scheduling, ReScheduling, and Open-Shop Scheduling problems", en Paper,

— Mason, S. J. (2005), "Heurística para minimizar la tardanza ponderada total en Job Shop complejos", en Paper

— Maus, R. y Keyes, J (1991), Handbook of Expert Systems in Manufacturing, CIUDAD, Mc Graw Hill.

- Méndez, G.A. (2001), Sistemas Cooperativos Asistidos para la Programación de la Producción en la Industria Manufacturera Colombiana, Bogotá D.C., Fondo de Publicaciones Universidad Distrital Francisco José de Caldas.

— Méndez, G.A. (2003), Gerencia de Manufactura, Bogotá D.C., Fondo de Publicaciones Universidad Distrital Francisco José de Caldas.
— Méndez, G. A. y Álvarez, L. (2004), Diseño de prototipo de diagnóstico para la pequeña y mediana empresa PYME. Enfoque mediante sistemas dinámicos, Bogotá D.C., Fondo de Publicaciones Universidad Distrital Francisco José de Caldas.

- MetaxiotisETAXIOTIS, ASKOUNIS y PSARRAS. "Expert Systems in Production Planning and Scheduling: A state-of-the-art survey". Paper. Journal of Intelligent Manufacturing. Atenas. Agosto de 2002.

— Mital, A. y Anand, S. (1994), Handbook of Expert Systems: Aplications in manufacturing, structures and rules, London, Champman \& Hall.

- Narasimhan, S.; Mcleavey, D. y Billington, M. (1996), Planeación de la producción y control de inventarios, México D.F., Prentice Hall.

— Pinedo, M. (2002), Scheduling Theory, Algorithms, and Systems, New Jersey, Prentice Hall.

- Reyes, C. (2005), "Herramientas de Software Inteligencia Artificial y Sistemas Expertos" [en línea], disponible en: http://www.monografias.com/trabajos26/sistemas-expertos/ sistemas-expertos.shtml\#desarr, recuperado: 1 de agosto de 2005 .

— Riley, G. () "CLIPS: A Tool for Building Expert Systems" [en línea], disponible en: http://www. ghg.net/clips/CLIPS.html, recuperado:

- Schroeder, R. (1992), Administración de operaciones, México D.F., Mc GrawHill.

- Subramaniam, V.; Raheja, A. y Bhupal, K. (2005), "Herramientas de reparación reactiva para secuenciación en Job Shop", en Paper

— Sule, D. y Joshi, R. () "Job Shop Scheduling with Parallel Processors”, en Paper

— Toal D.; Coffey, T. y Smith, P. (1994), "Expert Systems and Simulation in Scheduling", en Faltan datos. 
— Todd, D. y Sen, P. (1998), "Tackling complex Job Shop Problem using Operation Based Scheduling", en Paper

— Varela, R. () "Combinación de Algoritmos Evolutivos y Técnicas Heurísticas para Problemas de Scheduling", en Paper

— Vollman, T; Berry, W. y Whybark, C. (2000), Administración integral de la producción y de los inventarios, México D.F., Limusa Editores.

- Wallace, T. y Stahl, R. (2003), Master Scheduling in the $21^{\text {st }}$ Century, Cincinnati, Wallace \& Company.
- Wang, T. y Kuei, B.W. (2000), "Algoritmo de Enfriamiento Simulado revisado para obtener la mínima Tardanza Total en problemas de secuenciación tipo Job Shop", en Paper

— Wang, T. y Kuei, B.W. (2000), "Un Algoritmo Genético efectivo para secuenciación en $J o b$ Shop", en Paper

— "Sistemas Expertos" (en línea). Disponible en: http://www.esi2.us.es/ dco/sistemas.htm, Junio de 2006. 